\title{
ERRATUM
}

\section{Hydroxyl radical buffered by isoprene oxidation over tropical forests}

D. Taraborrelli, M. G. Lawrence, J. N. Crowley, T. J. Dillon, S. Gromov, C. B. M. Groß, L. Vereecken \& J. Lelieveld

Nature Geoscience 5, 190-193 (2012); published online 26 February 2012; corrected after print 28 February 2012.

This Letter was published online and in print with the author M. G. Lawrence being incorrectly affiliated to the Cyprus Institute. This has been corrected in the PDF and HTML versions of this Letter. 